\title{
Neutron Noise Analysis with Flash-Fourier Algorithm at the IBR-2M Reactor
}

\author{
Mihai O. Dima, ${ }^{1}$ Yuri N. Pepelyshev, ${ }^{2}$ and Lachin Tayibov ${ }^{2}$ \\ ${ }^{1}$ Institute for Physics and Nuclear Engineering, DFCTI, Magurele, 76900 Bucharest, Romania
}

${ }^{2}$ Joint Institute for Nuclear Research, FLNP, Dubna, Moscow 141980, Russia

Correspondence should be addressed to Mihai O. Dima; modima@nipne.ro

Received 19 September 2013; Accepted 17 November 2013

Academic Editor: Carlo Cattani

Copyright (C) 2013 Mihai O. Dima et al. This is an open access article distributed under the Creative Commons Attribution License, which permits unrestricted use, distribution, and reproduction in any medium, provided the original work is properly cited.

\begin{abstract}
Neutron noise spectra in nuclear reactors are a convolution of multiple effects. For the IBR-2M pulsed reactor (JINR, Dubna), one part of these is represented by the reactivities induced by the two moving auxiliary reflectors and another part of these by other sources that are moderately stable. The study of neutron noise involves, foremostly, knowing its frequency spectral distribution, hence Fourier transforms of the noise. Traditional methods compute the Fourier transform of the autocorrelation function. We show in the present study that this is neither natural nor real-time adapted, for both the autocorrelation function and the Fourier transform are highly CPU intensive. We present flash algorithms for processing the Fourier-like transforms of the noise spectra.
\end{abstract}

\section{Introduction}

Neutronic processes in nuclear reactors have a probabilistic character due to the quantum mechanics of scattering and the stochastic of propagation in materials. Design is mostly performed on the equations of neutron flux transport (in energy and space) and associated effects (fission, thermal fluxes, etc.). Statistical deviations from average quantities give however the complete image of neutron physics in the reactor-the so-termed neutron noise, described by large using Markovchain theories. The theories associated with the underlying stochasticity that produces the said fluctuations are actually century old $[1,2]$, stemming from population studies. It was thus shown that (Alphonse) de Candolle's conjecture on the extinction of family names [3-5] leads to nonergodic behavior in the case of neutron chains. Such theories were picked up in nuclear physics by Feynman, de Hoffmann and others to describe neutron processes in fission $[6,7]$, leading to the Feynman $\alpha$-formula:

$$
\frac{\sigma_{Z}^{2}(t)}{\langle Z(t)\rangle}=1+\epsilon\left(1-\frac{1-e^{-\alpha t}}{\alpha t}\right)
$$

which shows the fluctuations (1) being over Poissoniandue to correlations of neutrons in the same chain-and (2) the neutrons produced in the same group statistically disappearing (exponentially) all at the same time. The modern theoretical approach is given by the Pal-Bell equation [814], as an applied case of the Chapman-Kolmogorov master equation to Markov-chain neutron processes.

On top of said, neutron stochastic behavior is the modulation of the neutron flux by various reactivities: some due to 2-phase liquid flow (bubbling), or fuel embrittlement, mechanically induced reactivities, and so forth. Any addition to the spectrum may be detected and classified, issuing a specific warning. In this respect, neutron noise spectrum analysis is a very far reaching tool in nuclear safety.

To analyze the noise, usually a Fourier transform of the autocorrelation function is performed. This is basically the same as the square of the Fourier transformed signal. In practice, one also needs a time window, given by ad hoc apodisation functions. This is rather arbitrary and nonnatural. We show a more natural approach passing the noise through a resonant analyzing instrument together with flash algorithms for processing the Fourier-like transforms of the noise spectra. 


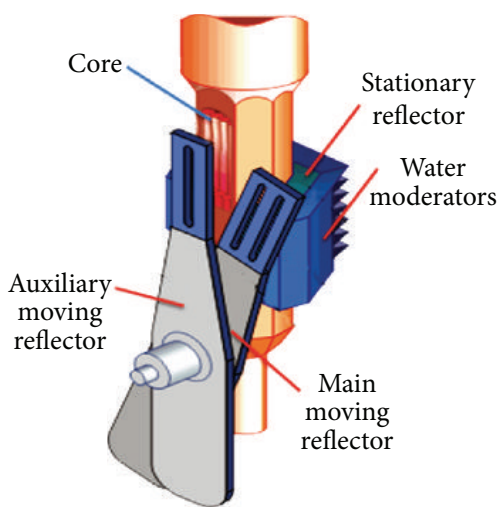

FIGURE 1: Details of the IBR-2M reactor showing the active core and the two movable reflectors.

\section{The IBR-2M Reactor}

The IBR-2M [15-17] is a modification of the IBR-2 (2 MW nominal power) pulsed research fast reactor with $\mathrm{PuO}_{2}$ fuel elements. The reactor coolant is liquid sodium. The pulsed mode operation is enabled by a reactivity modulator consisting of two rotating parts - a main movable reflector (OPO, at $1500 \mathrm{rpm}$ ) and an auxiliary movable reflector (DPO, at $300 \mathrm{rpm}$ ) - shown in Figure 1, that create reactivity pulses. For nominal DPO rotation speed, the reactivity of every fifth pulse is positive; that is, the reactor becomes prompt neutron supercritical for ca. $0.400 \mathrm{~ms}(0.215 \mathrm{~ms}$ half-width), with a repetition frequency of $5 \mathrm{~Hz}$ (Figure 2). As a result, powerful power pulses of $5 \mathrm{~Hz}$ repetition frequency take place in the reactor. The energy range of the fast neutrons is up to $10 \mathrm{MeV}$, with an almost linear logarithmic distribution versus energy.

The pulsed operation mode of the reactor is established when the prompt neutron supercriticality $(\delta k-\beta)$ reaches the "equilibrium" value $\varepsilon_{m}=\varepsilon_{m_{0}} \sim 1 \cdot 10^{-3}$ (at $5 \mathrm{~Hz}$ ) at which the reactor can be periodically pulsed. For supercriticality smaller than the "equilibrium" value, the amplitude (and consequently the energy) of each subsequent pulse is smaller than that of the previous one, which means that the reactor is attenuating. There are two main causes for pulse energy fluctuation in the reactor: the stochastic character of fission and neutron multiplication processes, and the fluctuation of external reactivity. Stochastic noise dominates power fluctuations at low neutron intensity for powers below $1 \mathrm{~W}$. Pulse energy fluctuations at high power have adverse effects on the operation of the reactor: in the dynamics, startup and adjustment process, performance of the experimental equipment, and so forth. However, power fluctuations also have a positive aspect, being as a tool for reactor diagnosis. The most important characteristic of a pulsed reactor is the relative dispersion of pulse energy fluctuations:

$$
\left(\frac{\sigma_{\mathrm{Q}}}{\overline{\mathrm{Q}}}\right)^{2}=\Delta_{\mathrm{st}}^{2}\left(1+\delta_{0}^{2}\right)+\delta_{0}^{2},
$$

where $\Delta_{\text {st }}^{2}=\nu \Gamma / 2 S \tau$ is the relative dispersion of stochastic fluctuations; $\delta_{0}^{2}$ is the relative dispersion of noise caused by external reactivity fluctuations; $\Gamma$ and $\nu$ are the dispersion

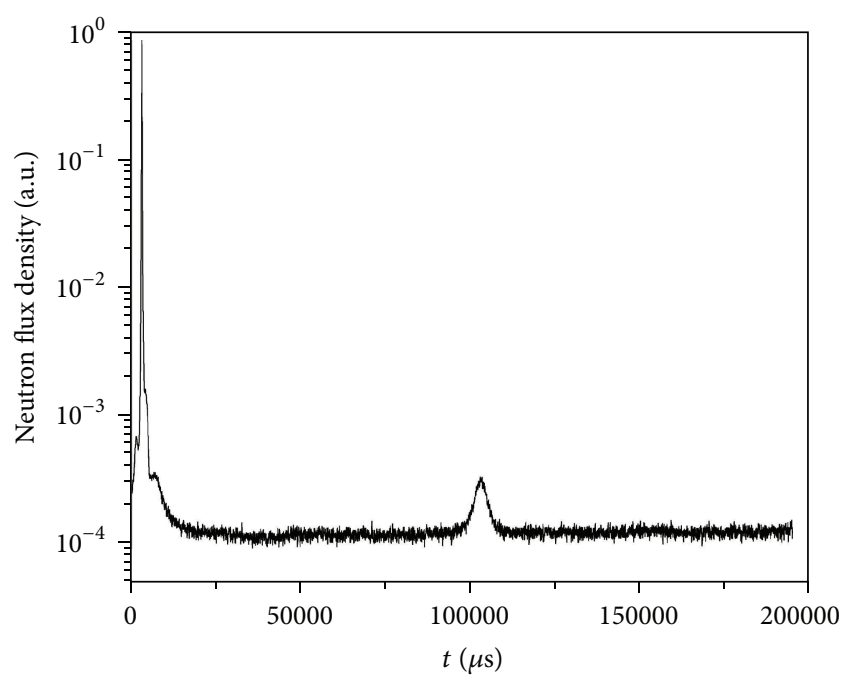

Figure 2: Power versus time between two subsequent pulses. Data is normalized to the maximum pulse.

of the number of prompt neutrons, and the average number of neutrons in a single fission event, respectively; $\tau$ is the lifetime of prompt neutrons in the core; $S=S_{\mathrm{sp}}+S_{\mathrm{del}}$ is the neutron source intensity (that operates continuously during the pulse); $S_{\mathrm{sp}}$ is the intensity of spontaneous neutrons and those from the $(\alpha, n)$ reaction on oxygen ${ }^{18} \mathrm{O}$ (part of the oxide fuel); $S_{\text {del }}=\beta_{\text {eff }} \nu F$ and $\beta_{\text {eff }}$ are the intensity, and effective fraction of delayed neutrons respectively; $F=L \cdot W$ is the fission rate; $L$ is the number of fission events in a second per watt; and $W$ is the absolute reactor power. At high power (for IBR-2M above $100 \mathrm{~W}$ ) the fluctuations of the external reactivity (component $\delta_{0}^{2}$ ) dominates. Basically, the mechanical vibrations of the two moving auxiliary reflectors cause this. All noise diagnostics of the reactor is based on research of this noise component, which naturally degrades in time. The power is dependent on the neutron flux; hence, monitoring reactor power indicates the size of the neutron flux, together with its fluctuations.

\section{Power Noise Baseline and Fourier Transform}

For a power noise analysis [18-21], we must first extract any reactor dynamics influences from the recorded waveforms (overcompensation by the AR power control rod, etc.). In this respect, we want to determine a baseline with respect to which reference the instantaneous power by subtraction obtains the power noise. Such method, generally for any power-generating systems, has been presented elsewhere [22]. The method applied here is a spline of third order over \pm 30 bins around each current point. Basically, the order of the spline and the number of bins were so determined such that the effective cut-off frequency is below the natural cut-off of the mechanical vibrations of the moving reflectors in our case $0.2 \mathrm{~Hz}$. The result is presented in Figure 3. 


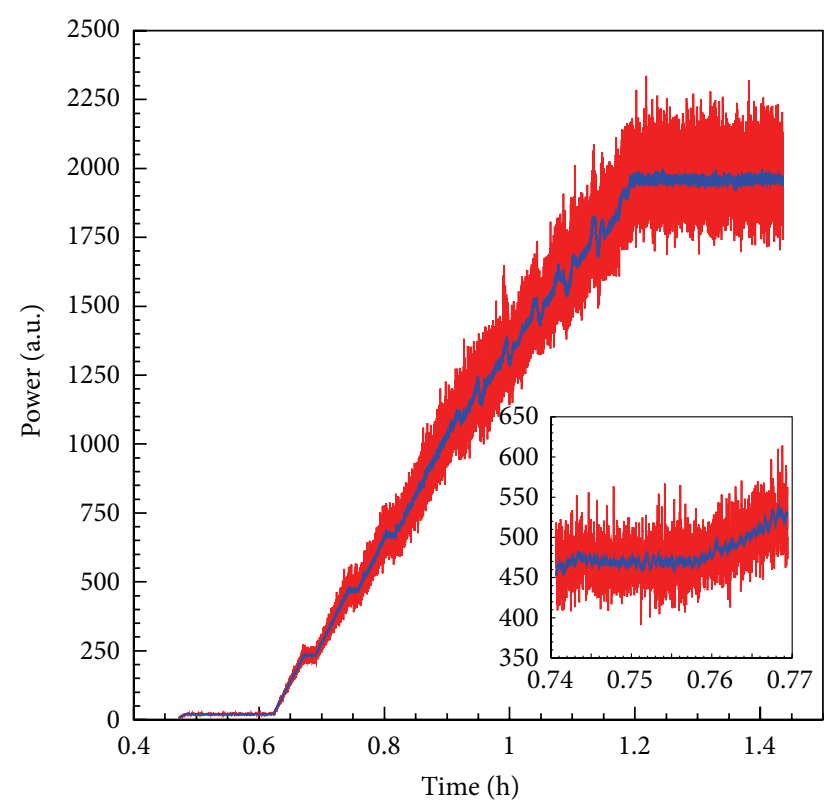

FIGURE 3: Reactor power during power up (red line) with fitted baseline (blue curve of spline order $k=3$ over a time interval $[-30,+30]$ bins).

The concept of Fourier transform in engineering can be quite different from its mathematical analogue. In mathematics, a given signal (function of time) is transformed to another function of frequency, basically the coefficients of the signal in the canonically conjugate image.

In engineering, a signal is long, and we need an "instantaneous" Fourier Transform at each given time, thus a function that provides a time-window-termed apodisation function. The question is, for discrete Fourier transforms (DFFT-[23]), how to perform said clipping, as the apodisation function introduces sidelobes to the central lobe describins the signalbest seen for the Parzen apodisation function, in Figure 4.

Traditionally, the Fourier transform of the power noise is computed from the autocorrelation function:

$$
P_{w} \sim V_{w}^{2}=\frac{1}{2 \pi} \int_{-\infty}^{+\infty} e^{i w \tau} r_{V}(\tau) d \tau,
$$

where $r_{V}(\tau)$ is the autocorrelation function:

$$
r_{V}(\tau)=\int_{-\infty}^{+\infty} V^{*}(t) V(t+\tau) d t .
$$

It is evident that although the Fourier transform is a single integral, the problem of calculating a double integral (high CPU cost), has not been eliminated, neither that of calculating the autocorrelation function.

Beyond the above considerations, special tools are needed to compute-what is mathematically improper termedFourier Transform of noise signals. The basic image is that of sound noise, perceived as higher or lower pitch. In categorizing noise as such, the ear for instance (as one example of analysis instrument) does not work with orthogonal wavevectors, or mathematically complete spaces, nor does it obtain coefficients for said wave-vectors. What such an analysis instrument does is to use a set of resonance phenomena, tuned on various frequencies. The instantaneous mechanical quantity producing measurable effect is (in the case of sound) the air velocity, or in electrical circuits, the current:

$$
\begin{gathered}
i=\frac{2 w_{0}}{R \sqrt{4 Q^{2}-1}} \int_{0}^{t} \epsilon(\tau) e^{w_{0}((\tau-t) / 2 Q)} \\
\times \sin \left(w_{0}(\tau-t) \sqrt{\left.1-\frac{1}{4 Q^{2}}-\phi\right) d \tau}\right. \\
\sin \phi=\sqrt{1-\frac{1}{4 Q^{2}}} \\
\cos \phi=\frac{-1}{2 Q}
\end{gathered}
$$

where $Q$ is the quality factor, $\omega_{0}$ the resonant pulsation, $R$ the electrical circuit's resistance, and $\varepsilon(t)$ is the driving voltage. It can be seen that the "analysis instrument" performs "something like" a Fourier Transform with an asymmetrical Poisson apodising function, looking into the "past."

As shown above, this is a natural choice-with a definite model in sight-for an apodising function, containing what is natural as analysis instrument.

The remaining problem is that such an instrument is highly computationally the intensive.

It can be recognized that the sine function can be written as sum of exponentials and that the mathematical construct to be computed is principally

$$
\int_{0}^{T} f(t) e^{i w t} d t=\sum f(n \Delta) e^{i w\left(n-n_{0}\right) \Delta} \cdot \Delta,
$$

where $\Delta$ is the bin size and $n$ the bin number over which summation occurs.

There are two cases for how the summation padding is advantageous, depending on how $\{2 \pi / \omega \Delta\}$ compares to $1 / 2$, where $\{\cdots\}$ and $[\cdots]$ are the fractional, respectively integer part:

(i) $\{2 \pi / \omega \Delta\} \geq 1 / 2$ : the sum at a given bin $n_{0}$ is

$$
S_{n_{0}}=\sum_{n=n_{0}}^{n_{0}+[2 \pi / w \Delta]} f(n \Delta) e^{i w \Delta\left(n-n_{0}+(1 / 2)\{2 \pi / w \Delta\}\right)} .
$$

The recurrence formula for obtaining the sum at the next point is obtained by shifting the exponentials with 1 bin, subtracting the lower end and adding the upper end:

$$
S_{n_{0}+1}=e^{-i w \Delta} S_{n_{0}}+f\left(n_{0}+1+\left[\frac{2 \pi}{w \Delta}\right]\right) e^{i \phi}-f\left(n_{0}\right) e^{-i \phi} \text {, }
$$

where $\phi=\omega \Delta / 2(2-\{2 \pi / \omega \Delta\})$. In doing so, most of the terms are known and can be computed in advance; the previous bin's sum is also known, so very little needs to be computed for the current bin. 


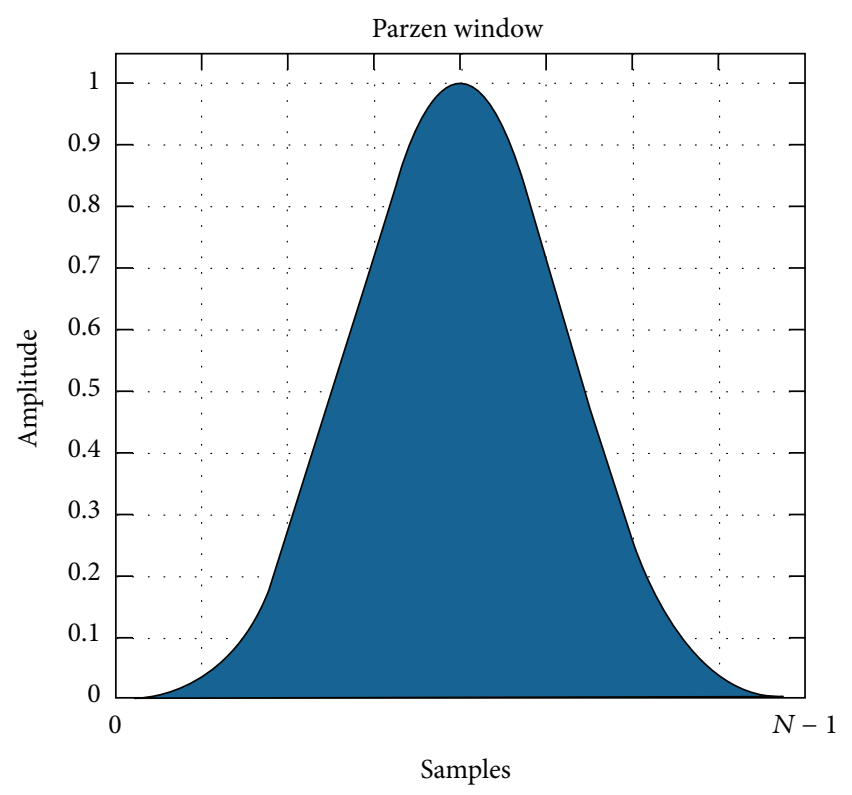

(a)

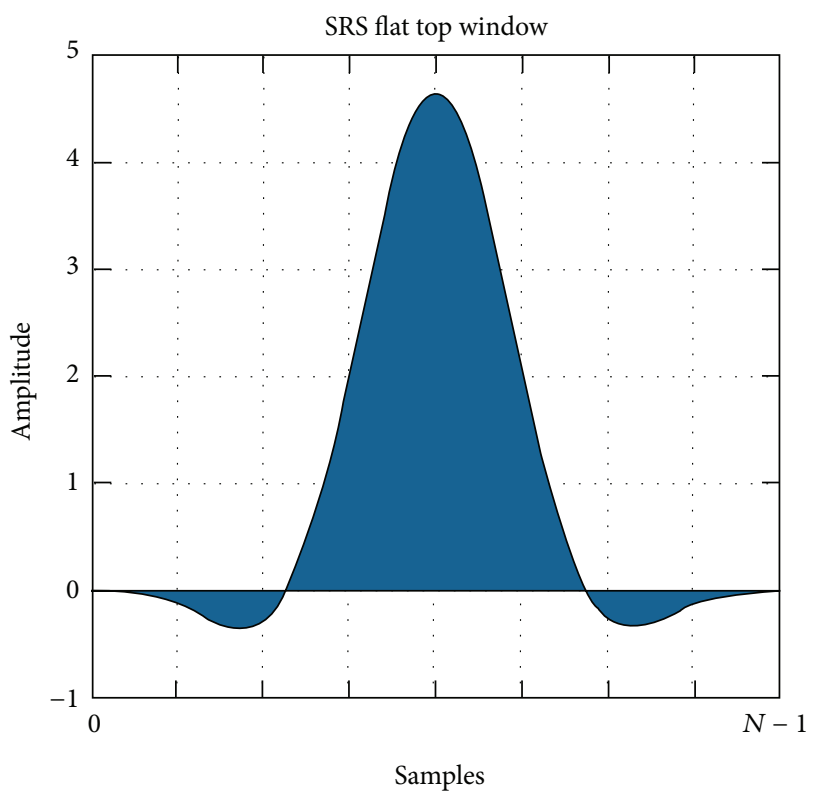

(c)

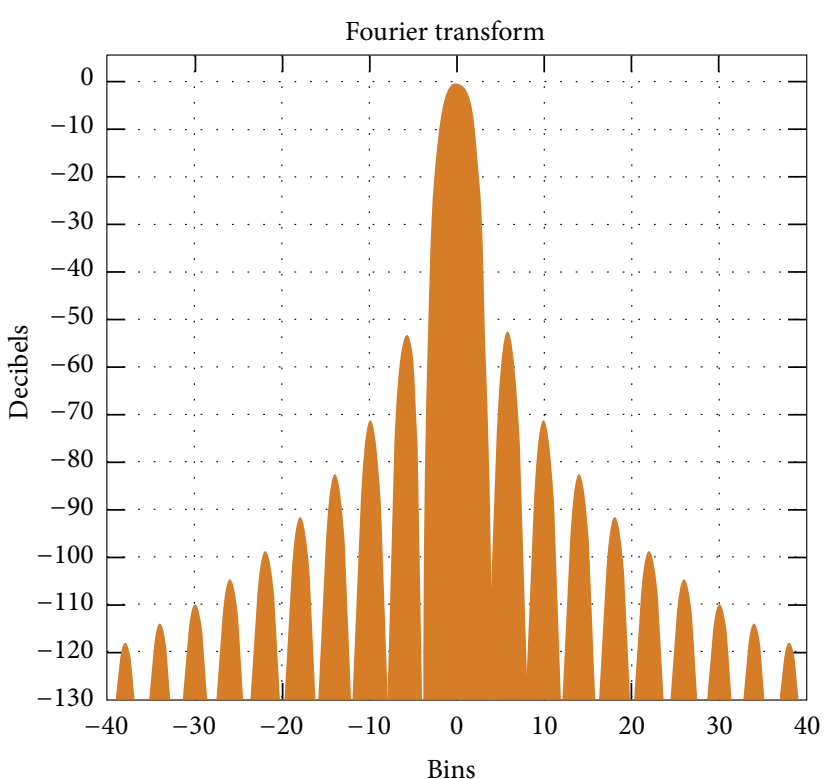

(b)

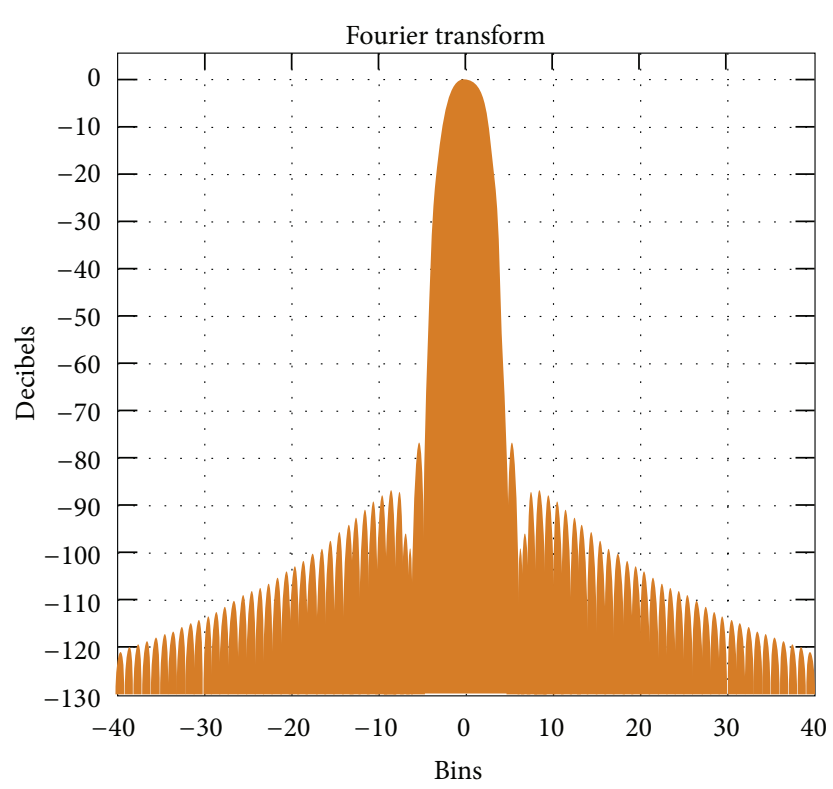

(d)

FIGURE 4: Fourier spectrum for various apodising functions. The Parzen function shows best the sidelobes that occur, while the SRS function provides a relatively flattop spectrum with good side-rejection of the lobes.

(ii) $\{2 \pi / \omega \Delta\}<1 / 2$ : the sum at a given bin $n_{0}$ is

$$
S_{n_{0}}=\sum_{n=n_{0}}^{n_{0}-1+[2 \pi / w \Delta]} f(n \Delta) e^{i w \Delta\left(n-n_{0}+1 / 2+(1 / 2)\{2 \pi / w \Delta\}\right)} .
$$

The recurrence formula for obtaining the sum at the next point is obtained by shifting the exponentials with 1 bin, subtracting the lower end and adding the upper end:

$$
S_{n_{0}+1}=e^{-i w \Delta} S_{n_{0}}+f\left(n_{0}+\left[\frac{2 \pi}{w \Delta}\right]\right) e^{i \phi}-f\left(n_{0}\right) e^{-i \phi}
$$

where $\phi=\omega \Delta / 2(1-\{2 \pi / \omega \Delta\})$. In doing so, most of the terms are known and can be computed in advance; the previous bin's sum is also known, so very little needs to be computed for the current bin.

As mentioned in the introduction, there are beneficial aspects in detecting and subtracting the baseline. Apart from extracting from the low frequency end reactor dynamics features, also technical aspects are achieved, such as rejecting aliasing. In general, such unwanted phenomenon is due to the fact that some frequency is much higher than the Nyquist 


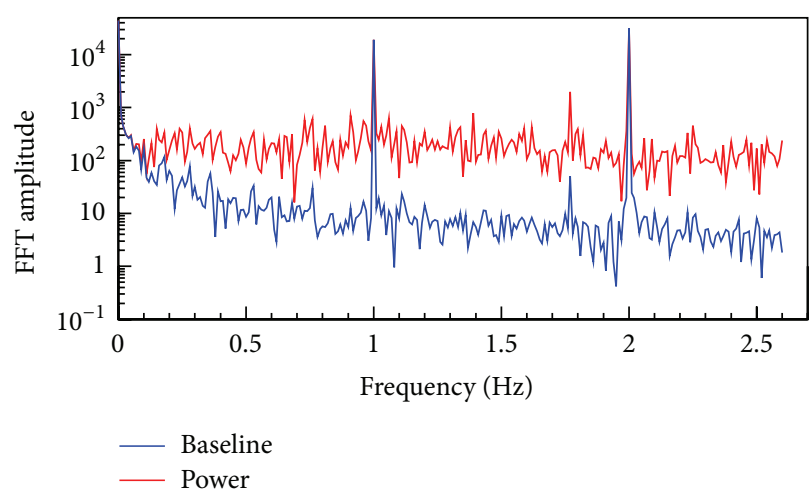

(a)

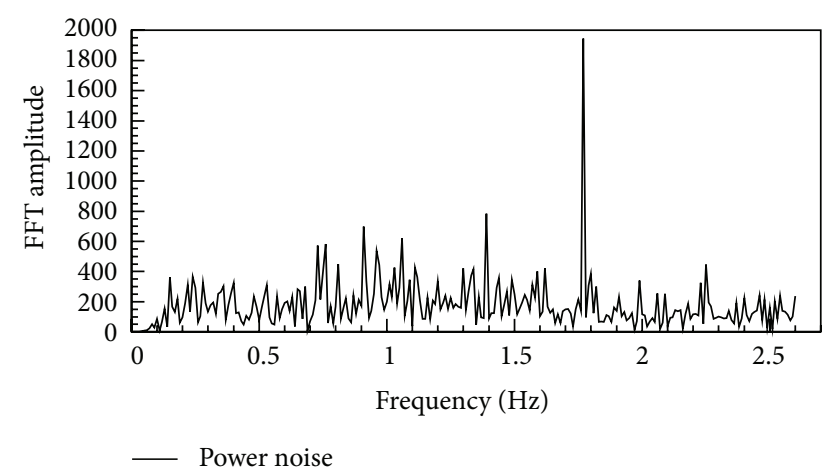

(b)

FIGURE 5: Fourier transform of reactor power, baseline, and noise. It can be seen that the raw power and baseline both are affected by aliasing in the same amount. The strong peaks at $1 \mathrm{~Hz}$ and $2 \mathrm{~Hz}$ disappear tracelessly in the difference (power noise spectrum), proving the efficiency of the method.

sampling frequency; in our case, $f_{\mathrm{Ny}}=1 / 2 \Delta t=2.5 \mathrm{~Hz}$. Imagine the effect of $50 \mathrm{~Hz}$ ripple for instance, or that of certain reactor chirps. Active analog filters would come to mind as first resource since they have a somewhat higher $Q$-factor than passive filters. (Passive filters in the range $[0,2.5 \mathrm{~Hz}]$ are too bulky in our view of a DAQ setup.) Still, even active filters do not have that high $Q$-factors, and if they do, $50 \mathrm{~Hz}$ sources do have a certain bandwidth, rendering very high$Q$ filters useless. As for reactor chirps, they are of broad bandwidth and impossible to filter. All these phenomena create unwanted aliasing that is impossible to remove in any traditional way. Fortunately, the effect is present for both raw data and for the baseline fit. Subtracting one from the other yields a clean noise signal reliable for physics studies of the reactor. Figure 5 shows the Fourier transform calculated with the above method for the reactor power and baseline and noise. It can be seen that the raw power and baseline both are affected by aliasing in the same amount. The strong peaks at $1 \mathrm{~Hz}$ and $2 \mathrm{~Hz}$ disappear tracelessly in the difference (the power noise spectrum), proving the efficiency of the method.

A toolkit with spectrum processing various utility codes has been created to help do all the steps required in a modular fashion.

\section{Conclusions}

For low frequency power noise monitoring, it is in certain contexts impossible to remove aliasing frequency components, presampling. Although aliasing collision is not present in the high end of the spectrum, close to $f_{\mathrm{Ny}}$-this is being a naturally free region-in the low region, the post-DAQ processing method presented here of raw-baseline subtraction is the only effective means of aliasing collision suppression. Such context appears for instance when the sensors delivering the data have saturation and blind-times after high pulses (for instance of radiation), acting as an effective sampling that is not avoidable through filters. Also, very low-pass filters are difficult to design for $2.5 \mathrm{~Hz}$ in the case presented. Digital filters need high sampling rates to avoid the exact condition that is being presented. Analog active filters have a high $Q$; thus, broadband signals have components that bypass them. Passive very low-pass filters are extremely bulky, as the inductors need to reach into thousands of $\mathrm{mH}$ range.

The method presented is feasible in real time, via a flash updating procedure of the data on the fly, allowing a physically credible estimate of noise spectral density.

It is interesting to note that FFT of noise is a very useful tool also in monitoring the electrical grid, for instance in observing hysteresis noise in transformer cores (leading to magnetic embrittlement and anomalous heating of the core, resp., transformer power losses). Other types of losses (dielectric polarization losses in areas with high humidity) can also be well monitored with this method.

Other nuclear reactor types could also benefit from the method, in principle power reactors which have significant water cooling systems, as these reactors produce vacuum reactivity noise if boiling occurs in the pipes.

\section{Acknowledgments}

One of the authors (M. Dima) acknowledges the support by a grant from the Romanian National Authority for Scientific Research ANCS Grant PN09370104 and two of the authors (Y. N. Pepelyshev and L. Tayibov) acknowledge the support by a grant from JINR, Dubna, Order No. 71, Item 21/2012.

\section{References}

[1] D. G. Kendall, "Branching processes since 1873," Journal of the London Mathematical Society, vol. 41, pp. 385-406, 1966.

[2] T. E. Harris, The Theory of Branching Processes, Springer, Berlin, Germany, 1963.

[3] A. de Candolle, Histoire des Sciences et des Savants, Genève: H. Georg, Paris, France, 1873.

[4] F. Galton, Problem 4001, The Educational Times, 1873.

[5] H. W. Watson and F. Galton, "On the probability of the extinction of families," The Journal of the Anthropological Institute of Great Britain and Ireland, vol. 4, p. 138, 1875. 
[6] R. P. Feynman, F. de Hoffmann, and R. Serber, "Dispersion of the neutron emission in U-235 fission," Journal of Nuclear Energy, vol. 3, no. 1-2, pp. 64-69, 1956.

[7] L. Pál and I. Pázsit, "Theory of neutron noise in a temporaly fluctuating multiplying medium," Nuclear Science and Engineering, vol. 155, pp. 425-440, 2007.

[8] I. Pázsit and L. Pál, Neutron Fluctuations: A Treatise on the Physics of Branching Processes, Elsevier, Amsterdam, The Netherlands, 2007.

[9] I. Pazsit and A. Enqvist, Neutron Noise in Zero Power Systems: A Primer in the Physics of Branching Processes, Chalmers University, Gothenburg, Sweden, 2008.

[10] F. Zinzani, C. Demazière, and C. Sunde, "Calculation of the eigenfunctions of the two-group neutron diffusion equation and application to modal decomposition of BWR instabilities," Annals of Nuclear Energy, vol. 35, no. 11, pp. 2109-2125, 2008.

[11] L. Pál and I. Pázsit, "Neutron fluctuations in a multiplying medium randomly varying in time," Physica Scripta, vol. 74, no. 1, pp. 62-70, 2006.

[12] I. Pázsit, Transport Theory and Stochastic Processes, Lecture Notes, Department of Nuclear Engineering, Chalmers University of Technology, Gothenburg, Sweden, 2007.

[13] I. Pázsit, C. Demaziere, C. Sunde, A. Hernandez-Solis, and P. Bernitt, "Final report on the research project ringhals diagnostics and monitoring, stage-12," Tech. Rep. CTH-RF-194/RR14, Chalmers University of Technology, Gothenburg, Sweden, 2008.

[14] C. Sunde, Noise diagnostics of stationary and non-stationary reactor processes [Ph.D. thesis], Department of Nuclear Engineering, Chalmers University of Technology, Gothenburg, Sweden, 2007.

[15] Y. N. Pepelyshev and A. K. Popov, "Investigation of dynamical reactivity effects of IBR-2 moving reflectors," Atomic Energy, vol. 101, no. 2, pp. 549-554, 2006.

[16] I. M. Frank and P. Pacher, "First experience on the high intensity pulsed reactor IBR-2," Physica B, vol. 120, no. 1-3, pp. 37-44, 1983.

[17] M. Makai, Z. Kalya, I. Nemes, I. Pos, and G. Por, "Evaluating new methods for direct measurement of the moderator temperature coefficient in nuclear power plants during normal operation," in Proceedings of the 17th Symposium of AER on VVER Reactor Physics and Reactor Safety, Yalta, Ukraine, September 2007.

[18] Y. N. Pepyolyshev, "Method of experimental estimation of the effective delayed neutron fraction and of the neutron generation lifetime in the IBR-2 pulsed reactor," Annals of Nuclear Energy, vol. 35, no. 7, pp. 1301-1305, 2008.

[19] W. Dzwinel, Y. N. Pepyolyshev, and K. Janiczak, "Predicting of slow noise and vibration spectra degradation in the IBR2 pulsed neutron source using a neural network simulator," Progress in Nuclear Energy, vol. 43, no. 1-4, pp. 145-150, 2003.

[20] W. Dzwinel, Pepyolyshev, and N. Yu, "Pattern recognition, neutral networks, genetic algorithms and high performance computing in nuclear reactor diagnostics-results and perspectives," in Proceedings of the 7th Symposium on Nuclear Reactor Surveillance and Diagnostics (SMORN '95), Avignon, France, June 1995.

[21] Y. N. Pepyolyshev and W. Dzwinel, "Pattern recognition application for surveillance of abnormal conditions in a nuclear reactor," Annals of Nuclear Energy, vol. 18, no. 3, pp. 117-123, 1991.
[22] M. O. Dima, Y. N. Pepelyshev, and T. Lachin, "Flash algorithms for power noise analysis at the IBR-2M Reactor," AIE, vol. 2, no. 1, pp. 21-25, 2014.

[23] J. O. Smith, Mathematics of the Discrete Fourier Transform (DFT) with Audio Applications, Center for Computer Research in Music and Acoustics (CCRMA), 2nd edition, 2007, http://ccrma.stanford.edu/ jos/mdft/. 

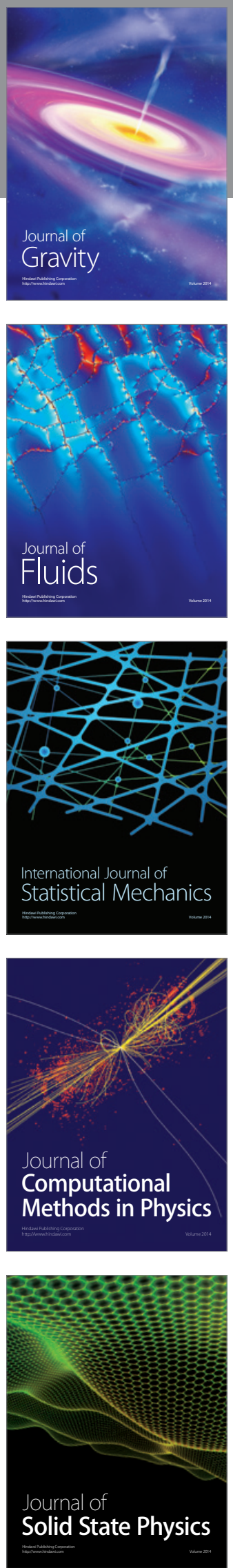

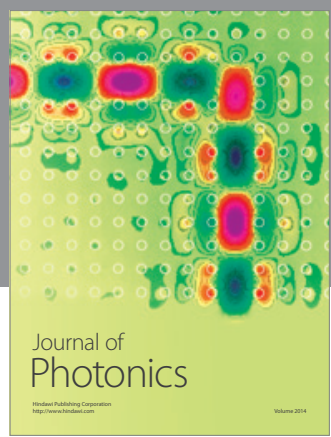

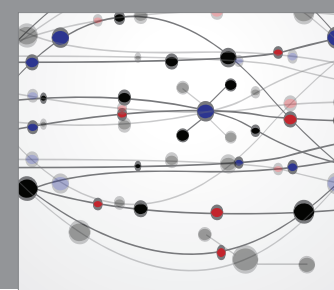

The Scientific World Journal

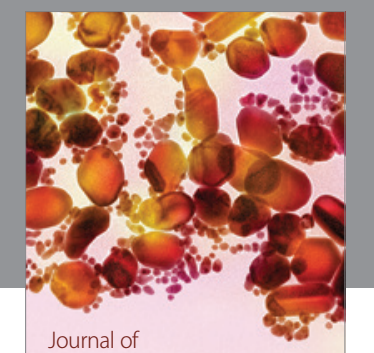

Soft Matter
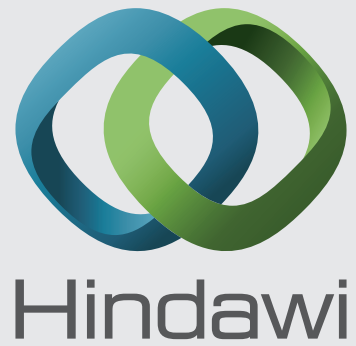

Submit your manuscripts at

http://www.hindawi.com
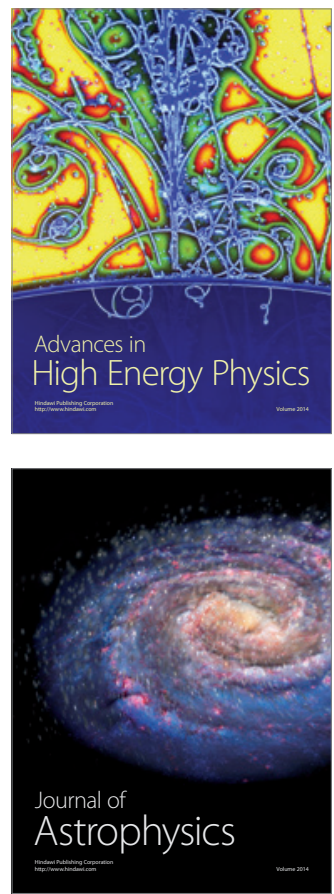
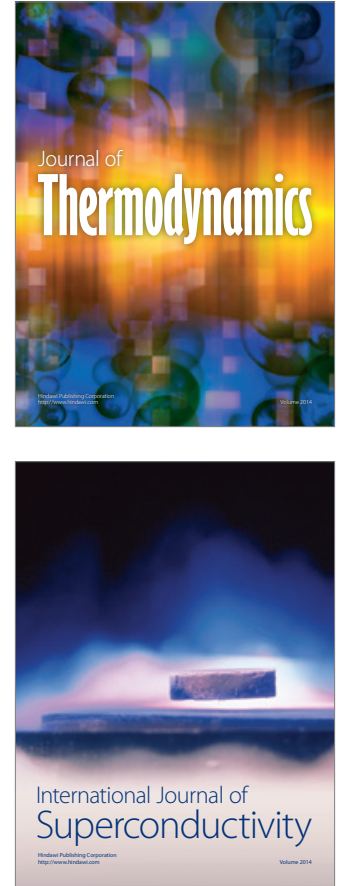
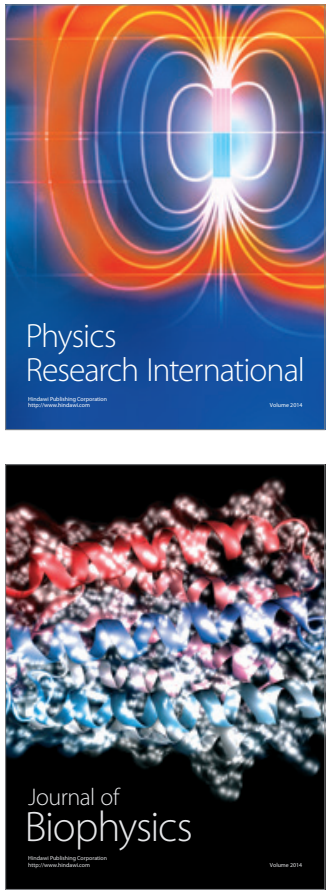
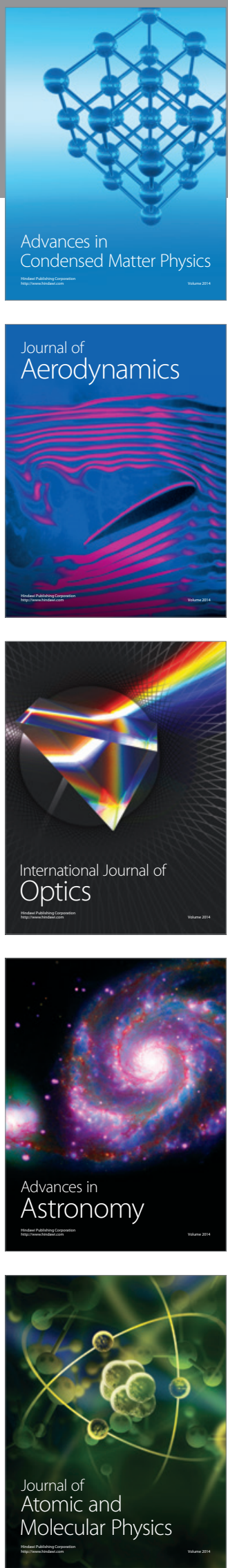